\title{
Feasibility of sacubitril/valsartan initiation early after acute decompensated heart failure
}

\author{
Agata Tymińska, Krzysztof Ozierański, Marcin Grabowski, \\ Grzegorz Opolski, Paweł Balsam \\ $1^{\text {st }}$ Department of Cardiology, Medical University of Warsaw, Poland
}

\begin{abstract}
Despite significant diagnostic and therapeutic advances, heart failure $(H F)$ is linked with high mortality and morbidity. Hospitalization for decompensated HF is still the most common cause of hospitalization in adults. What is more, a particularly high risk of hospitalization (even up to $50 \%$ of patients) is observed within a few months after a previous HF hospitalization. Sacubitril/valsartan, a first-in-class drug, contains a neprilysin inhibitor (sacubitril) and an angiotensin II receptor blocker (valsartan). In PARADIGM-HF trial investigators showed, that sacubitril/valsartan significantly reduced primary endpoint combined with cardiovascular death or HF hospitalization in patients with chronic, symptomatic HF (New York Heart Association class II-IV) with reduced ejection fraction (left ventricular ejection fraction $[L V E F] \leq 35-40 \%)$. Recently, results of the PIONEER-HF trial, which included HF patients with $L V E F \leq 40 \%$ who were hospitalized for acute decompensated HF were also published. The study proved that early, in-hospital, implementation of sacubitril/valsartan in these patients resulted in a substantially greater reduction of $N$-terminal prohormone $B$-type natriuretic peptide concentration and a lower rate of $H F$ rehospitalizations with similar safety profile for enalapril. (Cardiol J 2020; 27, 5: 625-632)
\end{abstract}

Key words: sacubitril/valsartan, acute decompensated heart failure, angiotensin receptor neprilysin inhibitor, angiotensin receptor neprilysin inhibitors (ARNI)

\section{Introduction}

Management of heart failure (HF) is one of the most important challenges of modern medicine in highly developed countries [1]. An aging population, effective invasive treatment of coronary artery disease and the advancement of new pharmacological molecules which improve the prognosis of patients with cardiovascular diseases could explain the increase in $\mathrm{HF}$ prevalence $[2,3]$. This is linked to the high costs of healthcare, which are mainly resulting from multiple hospitalizations due to worsening HF, as well as high mortality and poor quality of life (Fig. 1) [1, 3, 4].

Heart failure is a complex and progressive clinical syndrome caused by abnormalities of cardiac structure or function leading to inadequate cardiac output to fulfill metabolic demands or adequate cardiac output with increased left ventricular filling pressure [2]. There are multiple etiologies of HF, but it has been established that finally the same pathophysiological mechanisms are involved in the clinical progression of HF. The pathophysiology is based on progressive neurohormonal activation, involving two key systems: the renin-angiotensin-aldosterone system (RAAS) and the sympathetic nervous system (SNS). These mechanisms under physiological conditions are essential in the regulation of cardiovascular homeostasis in order to maintain proper cardiac function and perfusion of vital organs [5]. However, prolonged activation of these systems accelerates the progression of $\mathrm{HF}$ and promotes organ damage. Stimulation of the RAAS increases sodium and water retention, blood pressure, and also leads to fibrosis and remodeling of the myocardium and endothelial dysfunction with

Address for correspondence: Dr. Krzysztof Ozierański, $1^{\text {st }}$ Department of Cardiology, Medical University of Warsaw, ul. Banacha 1a, 02-097 Warszawa, Poland, tel: +48 2259929 58, e-mail: krzysztof.ozieranski@gmail.com 


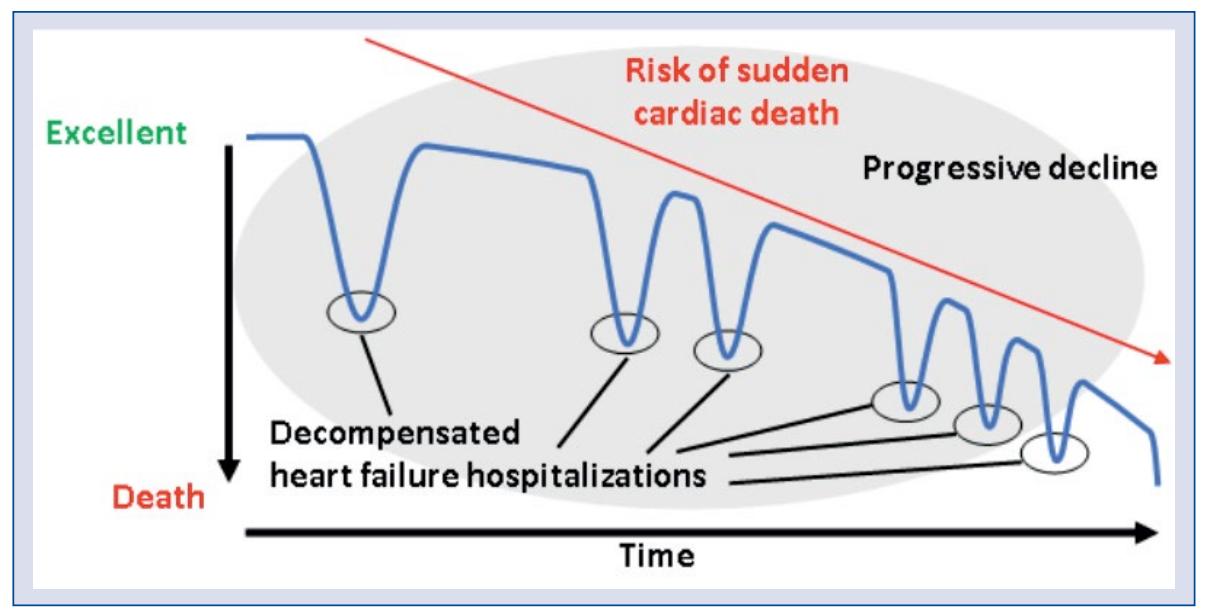

Figure 1. Clinical course of heart failure: progressive, chronic disease punctuated by acute episodes of exacerbation (based on and modified [29]).

the formation and destabilization of atherosclerotic plaques. Activation of the SNS results in vasoconstriction, increased heart rate and myocardial contractility [5].

In recent guidelines $\mathrm{HF}$ was classified into three subtypes - HF with reduced ejection fraction ( $\mathrm{HFrEF}), \mathrm{HF}$ with preserved ejection fraction (HFpEF), and HF mid-range ejection fraction (HFmrEF), according to the ejection fraction, natriuretic peptide levels and the presence of structural heart disease and diastolic dysfunction [2]. Differentiation of the HF subtype has important clinical and prognostic implications, as is a commonly accepted management with proven beneficial effects on prognosis, quality of life and acceptable safety profiles concerning patients with HFrEF [2]. However, patients with HFpEF and HFrEF have similarly high mortality risk and rate of rehospitalization after discharge $[6,7]$. There are currently two interesting on-going studies on patients with HFpEF - PARAGON-HF (Prospective comparison of Angiotensin Receptor-neprilysin inhibitor with ARB Global Outcomes in HF with preserved ejection fraction) and PARALLAX (A Randomized, Double-blind Controlled Study Comparing LCZ696 to Medical Therapy for Comorbidities in HFpEF Patients) will investigate the benefits of sacubtril/valsartan in patients with HFpEF $[8,9]$.

In this review, discussion focuses on the current role of sacubitril/valsartan in the management of patients with acute decompensated HFrEF, with particular regard to results from the PIONEER-HF and other recent studies.

\section{Current HFrEF treatment}

From almost two decades treatment of chronic HF have used angiotensin converting enzyme inhibitors (ACEI), angiotensin receptor blockers (ARB) and beta-blockers, followed by the implementation of mineralocorticoid receptor antagonists and ivabradine into clinical practice [2]. While symptomatic management is covered mainly by diuretics [2]. An important headway in the treatment of HFrEF in recent years was the development of a new drug containing a combination of valsartan and sacubitril, belonging to the angiotensin receptor neprilysin inhibitors (ARNI) [10].

In patients with acute decompensated HF the main part of management consists of improving patient signs and symptoms, correction of volume overload, improvement of hemodynamic status and counteracting the neurohormonal hyperactivation $[2,5]$. The key drugs in HF therapy in the acute setting are intravenous diuretics, vasodilators, and less commonly inotropic agents [2]. Nevertheless, despite rapid and aggressive initiation of therapy, long-term prognosis of patients with acute HF remain very poor. Therefore, there is a need for seeking for new and better therapeutic strategies to improve outcomes.

\section{Sacubitril/valsartan}

Sacubitril/valsartan is a first-in-class ARNI. This drug has a class I indication for treatment of symptomatic HFrEF in the current European and American guidelines $[2,11]$. The mechanism of 
action of this novel therapy includes RAAS inhibition through AT1 receptor blockade (valsartan) and neprilysin inhibiton (sacubitril), which increases levels of endogenous vasoactive peptides [12].

Besides the harmful activity of RAAS and SNS systems, other counter-regulatory pathways are activated in HF, including the natriuretic peptide (NP) system [13]. Sacubitril by inhibition of neprilysin reduces degradation of NP, bradykinin and other peptides. As a consequence, increased concentrations of mainly type A circulation (ANP) and type $\mathrm{B}$ natriuretic peptides (BNP) increases diuresis, natriuresis, and improves vasodilatation and relaxation of the myocardium. ANP and BNP also inhibits the secretion of renin and aldosterone. The selective blocking of the AT1 receptor reduces vasoconstriction, sodium and water retention and cardiac hypertrophy [6, 12-14].

\section{The PARADIGM-HF trial}

The PARADIGM-HF (Prospective Comparison of Angiotensin Receptor-Neprilysin Inhibitor with an Angiotensin-Converting Enzyme Inhibitor to Determine Impact on Global Mortality and Morbidity in Heart Failure) trial revealed that sacubitril/valsartan brings significant benefits among ambulatory patients with HFrEF compared with the use of RAAS inhibitor alone. Compared with enalapril, sacubitril/valsartan reduced by $20 \%$ the composite endpoint of cardiovascular death or $\mathrm{HF}$ hospitalization, giving a real chance for further improvement in HF therapy. Because of these results, the trial was stopped early after a median follow up of 27 months $[10,15]$. In consequence, sacubitril/ /valsartan received a strong recommendation in the European and American guidelines as an alternative for ambulatory $\mathrm{HFrEF}$ patients who tolerate an ACEI or ARB and are still symptomatic $[2,11]$.

\section{The PIONEER-HF trial — study design}

There is limited data on sacubitril/valsartan in an acute setting, such as in patients hospitalized for acute decompensated $\mathrm{HF}$ and patients with severe symptomatic chronic HF. However, it seems reasonable to initiate and intensify lifesaving chronic therapy already in the hospital to decrease the risk of premature $\mathrm{HF}$ re-exacerbation. The goal of the PIONEER-HF (Comparison of Sacubitril/Valsartan versus Enalapril on Effect on NT-proBNP in $\mathrm{Pa}$ tients Stabilized from an Acute Heart Failure Episode) trial was to assess the safety and efficacy of sacubitril/valsartan use in hospitalized individuals with acute decompensated HFrEF [16]. Interestingly, the PIONEER-HF trial was construed early after the appearance of positive data from the PARADIGM-HF study. As a rationale for conducting this trial, the researchers highlighted that in the PARADIGM-HF approximately $40 \%$ of participants had no previous HF hospitalization, and at most $15 \%$ of patients were hospitalized for a primary diagnosis of HF during the entire study [15]. What is more, patients with actual acute decompensated HF were excluded from the PARADIGM-HF study and only less than $1 \%$ of patients had New York Heart Association (NYHA) class IV symptoms at baseline [15].

In the PIONEER-HF trial 881 patients were recruited with HFrEF ( $\leq 40 \%)$, currently hospitalized for acute decompensated HF with elevated NP levels (N-terminal pro-B-type natriuretic peptide $[\mathrm{NT}$-proBNP] $\geq 1600 \mathrm{pg} / \mathrm{mL}$ or $\mathrm{BNP} \geq 400 \mathrm{pg} / \mathrm{mL}$ ). The randomization was not earlier than $24 \mathrm{~h}$ and up to 10 days from hospital admission. The patients had to be clinically stable. Inclusion and exclusion criteria from the PIONEER study are presented in Table 1 . After achieving hemodynamic stabilization, the patients were randomized $1: 1$ to sacubitril/ $/$ valsartan $(\mathrm{n}=440)$ or enalapril $(\mathrm{n}=441)$, and were then followed for 8 weeks. Initial dose of sacubitril/valsartan was $24 / 26$ or $49 / 51 \mathrm{mg}$, and for enalapril was 2.5 or $5 \mathrm{mg}$, both given twice daily. If the conversion was made from ACEI, there was a 36-h wash-out period. The investigators aimed to up-titrate the dose of sacubitril/valsartan to $97 / 103 \mathrm{mg}$ and enalapril to $10 \mathrm{mg}$ twice daily. Finally, they selected a surrogate biomarker (NT-proBNP) as the primary endpoint. The primary efficacy outcome was a change in NT-proBNP concentration from baseline to week 4 and week 8 [16]. Secondary efficacy and safety outcomes are listed in Table 2.

\section{Results of the PIONEER-HF trial}

In the PIONEER-HF trial mean age of patients was 61 years, $72 \%$ were male and $36 \%$ were black. At randomization, the median systolic blood pressure was $118 \mathrm{mmHg}$, and NT-proBNP concentration at screening was $4812 \mathrm{pg} / \mathrm{mL}$. The median left ventricular ejection fraction (LVEF) was $24 \%$; two thirds of patients had NYHA class III, and approximately $10 \%$ had NYHA class IV. The median serum creatinine was $1.3 \mathrm{mg} / \mathrm{dL}$; and serum potassium was $4.2 \mathrm{mmol} / \mathrm{L}$. Further, approximately two thirds of patients had previously beem diagnosed with $\mathrm{HF}$ and $60 \%$ had at least one HF hospitalization within 
Table 1. Inclusion and exclusion criteria for the PIONEER-HF trial (based on [16]).

\begin{tabular}{l} 
Inclusion criteria \\
\hline Adults $>18$ years of age with the capacity to \\
provide written informed consent \\
Currently hospitalized for acute decompensated HF \\
with symptoms and signs of fluid overload \\
LVEF $\leq 40 \%$ within the past 6 months \\
Elevated NT-proBNP $\geq 1600 \mathrm{pg} / \mathrm{mL}$ or BNP \\
$\geq 400 \mathrm{pg} / \mathrm{mL}$ during current hospitalization \\
Randomization not earlier than $24 \mathrm{~h}$ and up to \\
10 days from hospital admission \\
Systolic blood pressure $\geq 100 \mathrm{mmHg}$ for the \\
preceding $6 \mathrm{~h}$ before randomization and absence \\
of symptomatic hypotension \\
No increase (intensification) in intravenous diuretic \\
dose within the last $6 \mathrm{~h}$ prior to randomization \\
No use of intravenous vasodilators within $6 \mathrm{~h}$ prior \\
to randomization \\
No intravenous inotropic drugs $24 \mathrm{~h}$ prior \\
to randomization
\end{tabular}

Exclusion criteria

Currently taking sacubitril/valsartan or any use within the past 30 days

History of hypersensitivity, known or suspected contraindications, or intolerance to any of the study drugs, including ACEI, ARB, or sacubitril

Patients with a known history of angioedema related to previous ACEI or ARB therapy

Requirement of treatment with both $A C E I$ and $A R B$

Estimated glomerular filtration rate

$<30 \mathrm{~mL} / \mathrm{min} / 1.73 \mathrm{~m}^{2}$

Serum potassium $>5.2 \mathrm{mEq} / \mathrm{L}$

Known hepatic impairment or history of cirrhosis with evidence of portal hypertension

Acute coronary syndrome, stroke, TIA; cardiac, carotid, or other major cardiovascular surgery; percutaneous coronary intervention or carotid angioplasty, within the prior month

Implantation of cardiac resynchronization therapy within the past 3 months or intent to place Isolated right HF due to severe pulmonary disease Documented untreated ventricular arrhythmia with syncopal episodes within the past 3 months

Presence of hemodynamically significant mitral, aortic, or hypertrophic obstructive cardiomyopathy History of malignancy of any organ system (other than localized and resectable skin cancers) within the past year with a life expectancy of less than 1 year

Pregnant or nursing (lactating) women

ACEI - angiotensin converting enzyme inhibitors; ARB - angiotensin receptor blockers; BNP - type B natriuretic peptides; HF heart failure; LVEF — left ventricular ejection fraction; NT-proBNP - N-terminal pro-B-type natriuretic peptide; TIA — transient ischemic attack
Table 2. PIONEER-HF study end-points (based on [16]).

\begin{tabular}{l}
\hline Primary outcome \\
\hline Time-averaged proportional change in NT-proBNP \\
concentration [time frame: baseline, week 4 \\
and week 8] \\
\hline Secondary efficacy and safety outcomes \\
\hline Key safety outcomes \\
Number of patients with incidences of: \\
- symptomatic hypotension \\
- worsening renal function \\
- hyperkalemia \\
- angioedema \\
Secondary biomarkers outcomes \\
Change from baseline in: \\
- high sensitivity troponin T concentration \\
- BNP concentration \\
- ratio of BNP to NT-proBNP \\
Clinical outcomes: \\
Time to first occurrence of composite of \\
I. Death \\
II. Hospitalization for worsening HF \\
III. Left ventricular assist device implantation \\
IV. Listed for cardiac transplantation \\
V. Unplanned visit for acute HF requiring \\
intravenous diuretics \\
VI. Increase in diuretic dose $>50 \%$ \\
VII. Use of an additional drug for HF \\
\hline BNP- B-terminal pro-B-type natriuretic peptide \\
-
\end{tabular}

the previous year. At the time of randomization $61.7 \%$ of the patients had peripheral edema, $32.9 \%$ had rales on lungs auscultation and $93.0 \%$ received intravenous furosemide during the index hospitalization before randomization. Fifty-two percent of the patients were not receiving an ACEI or ARB at the time of hospital admission [16].

In the PIONEER-HF treatment with sacubitril/valsartan was associated with a greater time-averaged reduction in NT-proBNP concentration (primary efficacy outcome) compared to enalapril. The investigators also noted a $25.3 \%$ and $46.7 \%$ reduction in NT-proBNP concentration in enalapril and sacubitril/valsartan groups, respectively. This reduction was observed within the first week after drug initiation [16]. NT-proBNP is a biomarker of neurohormonal activation and hemodynamic stress, which plays an important role as a tool for 
HF diagnosis, monitoring of therapy and prognosis. It is worth noting, that HFrEF patients with concomitant atrial fibrillation (AF) have higher concentration of NT-proBNP. However, Kristensen et al. [17] showed that NT-proBNP $>400 \mathrm{pg} / \mathrm{mL}$ in those patients had a similar value in the prediction of cardiovascular outcomes comparing to $\mathrm{HF}$ patients without AF $[17,18]$.

The PIONEER-HF study also observed a reduction in high-sensitive troponin $T$ concentration in the sacubitril/valsartan group ( $<<0.05)$. Elevation of troponin is a very frequent finding in patients hospitalized for acute decompensated $\mathrm{HF}$ and is associated with poor outcomes during hospitalization and increased risk of death or rehospitalizations after discharge [19]. Nakou et al. [20] already showed that troponin I concentrations may be an independent predictive marker of a sacubitril/ /valsartan positive response in HFrEF.

Importantly, the PIONEER-HF study also showed a $44 \%$ reduction in HF rehospitalizations and $46 \%$ reduction in a composite outcome of serious clinical events (death, HF rehospitalization, need for a left ventricular assist device, or heart transplant). What is more, previously, Desai et al. [21] showed that patients treated with sacubitril/valsartan comparing to enalapril in the PARADIGM-HF study had less frequent 30-day readmissions for any cause after HF hospitalization. The results of these studies encourages early use of sacubitril/valsartan and gives an opportunity for additional improvement of outcomes of HF patients compared to enalapril. The results of the clinical and safety outcomes of the PIONEER-HF study are presented in Tables 3 and 4.

In the PIONEER-HF study, patients hospitalized for acute coronary syndrome with concomitant signs of HF were excluded from the study. However, an on-going PARADISE-MI (Prospective ARNI vs. ACE Inhibitor Trial to DetermIne Superiority in Reducing Heart Failure Events After MI) study enrolls patients with LVEF $<40 \%$, and signs of $\mathrm{HF}$ in the post-acute myocardial infarction phase (without prior chronic HF). The PARADISE-MI study was designed to evaluate benefits of sacubitril/valsartan versus ramipril in reducing the occurrence of composite endpoint of cardiovascular death, HF hospitalization and outpatient HF occurrence in patients with new-onset $\mathrm{HF}$ after recent myocardial infarction [22].

There are also other studies evaluating the process of initiation and uptitration of sacubitril/ /valsartan following hospitalization for acute decompensated HF. The rationale of the TRANSI-
TION (The Comparison of Pre- and Post-discharge Initiation of LCZ696 Therapy in HFrEF Patients After an Acute Decompensation Event) study was to evaluate efficacy and safety of in-hospital initiation of sacubitril/valsartan in HFrEF patients hospitalized for acute decompensated HF after clinical stabilization [23]. According to the protocol, patients were randomized within $\geq 24 \mathrm{~h}$ after hemodynamic stabilization (in a pre-discharge arm) or up to 14 days after discharge (a post-discharge arm). The study enrolled patients with deterioration of chronic HF or with de novo acute decompensated $\mathrm{HF}$, as well as patients with or without previous ACEI/ARB therapy [24]. The primary results of the TRANSITION study demonstrated that uptitration of sacubitril/valsartan to a target dose $200 \mathrm{mg}$ (sacubitril $97 \mathrm{mg}$ and valsartan $103 \mathrm{mg}$ twice daily) was achieved in about $45 \%$ of patients who started taking the drug before discharge, compared with $50 \%$ of patients who started the drug after discharge. The difference was not statistically significant. Adverse events prompting discontinuations of sacubitril/valsartan therapy were rare, and occurred similarly in both arms of the trial [24].

The PARADIGM-HF study recruited patients who were pre-exposed to optimal doses of enalapril (10 mg twice daily) and were then transitioned to sacubitril/valsartan (first $100 \mathrm{mg}$ (sacubitril $49 \mathrm{mg}$ and valsartan $51 \mathrm{mg}$ ), twice daily, and then sacubitril/valsartan $200 \mathrm{mg}$ (sacubitril $97 \mathrm{mg}$ and valsartan $103 \mathrm{mg}$ ), twice daily, over a 6-8 week period before randomization. In comparison, the TITRATION (Safety and Tolerability of Initiating LCZ696 in Heart Failure Patients) study was addressed to evaluate the tolerability of initiation/ /faster uptitration (condensed shorter 3-week and conservative 6 -week uptitration) of sacubitril/valsartan in $\mathrm{HF}$ patients with $\mathrm{LVEF} \leq 35 \%$. The study population was comprised of 498 in- and outpatients, both patients pre-exposed to varying doses of an ACEI/ARB and ACEI/ARB-naive. Initially, patients were taking $50 \mathrm{mg}$ sacubitril/valsartan twice a day for 5 days. The authors showed that sacubitril/valsartan was characterized by a good safety profile and tolerance regardless of time to reach the target dose. There were no statistically significant differences in the occurrence of hypotension, renal dysfunction, hyperkalemia and angioedema between 'condensed' vs. 'conservative' regimens. The secondary tolerability outcome was related to the number of patients who managed to reach the target dose $97 / 103 \mathrm{mg}$ twice daily and to maintain it for 12 weeks. Such therapeutic success was achieved in $75.9 \%$ of the study participants 
Table 3. Clinical and biomarker outcomes in the PIONEER-HF trial (based on [16]).

\begin{tabular}{|c|c|c|c|}
\hline Clinical outcomes & $\begin{array}{l}\text { Sacubitril/ } \\
\text { /valsartan } \\
(n=440)\end{array}$ & $\begin{array}{l}\text { Enalapril } \\
(\mathrm{n}=441)\end{array}$ & $\begin{array}{l}\text { Hazard ratio } \\
(95 \% \mathrm{Cl})\end{array}$ \\
\hline Composite of clinical events & $249(56.6 \%)$ & $264(59.9 \%)$ & $0.93(0.78-1.10)$ \\
\hline Death & $10(2.3 \%)$ & $15(3.4 \%)$ & $0.66(0.30-1.48)$ \\
\hline Rehospitalization for HF & $35(8.0 \%)$ & $61(13.8 \%)$ & $0.56(0.37-0.84)$ \\
\hline Implantation of left ventricular assist device & $1(0.2 \%)$ & $1(0.2 \%)$ & $0.99(0.06-15.97)$ \\
\hline Inclusion on the list for heart transplantation & $0(0 \%)$ & $0(0 \%)$ & NA \\
\hline $\begin{array}{l}\text { Unplanned outpatient visit leading to use } \\
\text { of intravenous diuretics }\end{array}$ & $2(0.5 \%)$ & $2(0.5 \%)$ & $1.00(0.14-7.07)$ \\
\hline Use of additional drug for HF & $78(17.7 \%)$ & $84(19.0 \%)$ & $0.92(0.67-1.25)$ \\
\hline Increase in dose of diuretics of $>50 \%$ & $218(49.5 \%)$ & $222(50.3 \%)$ & $0.98(0.81-1.18)$ \\
\hline $\begin{array}{l}\text { Composite of serious clinical events (death, } \\
\text { rehospitalization for HF, implantation of a left } \\
\text { ventricular device, inclusion on the list of patients } \\
\text { eligible for heart transplantation) }\end{array}$ & $41(9.3 \%)$ & $74(16.8 \%)$ & $0.54(0.37-0.79)$ \\
\hline Secondary biomarker outcomes & & & $\begin{array}{l}\text { Ratio of change } \\
(95 \% \mathrm{Cl})\end{array}$ \\
\hline $\begin{array}{l}\text { Change in high-sensitivity troponin T } \\
\text { concentration }\end{array}$ & \multicolumn{2}{|c|}{$-36.6(-40.8$ to -32.0$)-25.2(-30.2$ to -19.9$)$} & $0.85(0.77-0.94)$ \\
\hline Change in BNP concentration & \multicolumn{2}{|c|}{-28.7 ( -35.5 to -21.3$)-33.1$ ( -39.5 to -25.9$)$} & $1.07(0.92-1.23)$ \\
\hline Change in ratio of BNP to NT-proBNP & 35.2 (28.8 to 42.0 ) & $-8.3(-3.6$ to -12.7$)$ & $1.48(1.38-1.58)$ \\
\hline
\end{tabular}

BNP - B-type natriuretic peptide; $\mathrm{Cl}$ - confidence interval; HF — heart failure; NA - not available, NT-proBNP - N-terminal pro-B-type natriuretic peptide

Table 4. Safety outcomes in the PIONEER-HF trial (based on [16]).

\begin{tabular}{lccc}
\hline Safety outcome & Sacubitril/valsartan $(\mathbf{n}=440)$ & Enalapril $(\mathbf{n}=441)$ & Relative risk (95\% CI) \\
\hline Worsening renal function* & $60(13.6 \%)$ & $65(14.7 \%)$ & $0.93(0.67-1.28)$ \\
Hyperkalemia & $51(11.6 \%)$ & $41(9.3 \%)$ & $1.25(0.84-1.84)$ \\
Symptomatic hypotension & $66(15.0 \%)$ & $56(12.7 \%)$ & $1.18(0.85-1.64)$ \\
Angioedema & $1(0.2 \%)$ & $6(1.4 \%)$ & $0.17(0.02-1.38)$ \\
\hline
\end{tabular}

*Worsening renal function was defined by an increase in the serum creatinine concentration of $0.5 \mathrm{mg}$ per deciliter or more ( $\geq 44 \mu \mathrm{mol} / \mathrm{L})$ and a decrease in the estimated glomerular filtration rate of $25 \%$ or more; $\mathrm{Cl}$ - confidence interval

(in $78 \%$ of people in the 3 -week group and $84 \%$ in the 6 -week group, $\mathrm{p}=0.07$ ). It may be concluded, based on the results of the TITRATION study that initiation/uptitration of sacubitril/valsartan from 50 to $200 \mathrm{mg}$ twice daily had a good tolerability over the 3 - and 6 -week process, but more gradual uptitration may have fewer side effects and may be better tolerated in patients previously treated with low doses of ACEI or ARB (or ACEI/ARB naive) [25].

What is highly important in terms of drug initiation in the acute setting, was that the PIONEER-HF trial sacubitril/valsartan was well tolerated and showed a good safety profile. Rates of the key safety outcomes including symptomatic hypotension, worsening renal function, hyperkalemia, or angioedema were comparable between the two study arms (for all p-value $>0.05$ ). In addition, all 6 cases of angioedema in the enalapril group occurred in black patients, while the only case in the sacubitril/valsartan group was in a white patient. Trial medication was discontinued in approximately $20 \%$ of patients in both groups [16]. In contrast, in the PARADIGM-HF study, hypotension was more frequent in the sacubitril/valsartan group, while hyperkalemia, higher serum creatinine level, need for discontinuation of the study drug because of renal impairment and cough were more common in the enalapril group (for all p-value $>0.05$ ). Sacubitril/ /valsartan was discontinued in $17.8 \%$ and enalapril in $19.8 \%$ of patients $(\mathrm{p}=0.02)$ [15]. 
Luo et al. [26] assumed that implementation of the novel therapy with ARNI into the clinical practice is slow and they were seeking characteristics of early adopters and factors associated with ARNI prescription among patients discharged after acute HF hospitalization. They analyzed 16674 HFrEF patients hospitalized in 210 hospitals from October 2015 to December 2016. ARNI was prescribed at discharge for $6.1 \%$ of them. They showed that for-profit hospitals located in the Northern United States had significantly higher odds of ARNI prescription compared with not-for-profit hospitals located in the Western United States ( $p=0.04$ and $p=0.02$, respectively) [26]. Further studies assessing sacubitril/valsartan will perhaps translate into a better understanding of the new evidence-based therapy and minimize differences across hospitals.

New evidence regarding the use of sacubitril/ /valsartan in patients hospitalized for new-onset HF or decompensated chronic HF sacubitril/valsartan was included in the European Society of Cardiology 2019 experts' clinical practice update on HF [27]. According to this new document sacubitril/valsartan, rather than an ACEI or an ARB, may be considered in these patients to reduce short-term risk of adverse outcomes. The direct introduction of sacubitril/valsartan, without the need of overtaking ACEI titration, significantly facilitates management of $\mathrm{HF}$ patients.

Authors of the PIONEER-HF trail pointed out some limitations of the study. They concluded that there was a need to wait for hemodynamic stability and a 36-h wash-out period in the sacubitril/ /valsartan group, with $6 \mathrm{~h}$ of obligatory observation, may require prolonged hospital stays. However, the median duration of the index hospitalization (5.2 days) was shorter than was shown in a previous analysis from the registry of European Society of Cardiology (median hospital stay was 7 days) [28]. Additionally, there was high discontinuation rate of study drug in both arms and 15\% had missing data for the primary endpoint [16].

\section{Conclusions}

The results of the presented studies encourage the early initiation of sacubitril/valsartan treatment immediately after achieving clinical stabilization to improve outcomes of HF patients after hospitalization for worsening HF. Treatment of clinically stabilized HFrEF patients hospitalized for acute decompensated HF with sacubitril/ /valsartan significantly reduces NT-proBNP con- centrations and the risk of serious clinical events. An early start of treatment with sacubitril/valsartan has a good safety profile and is not associated with an increased risk of symptomatic hypotension, renal dysfunction, hyperkalemia, or episode of angioedema compared to enalapril.

Conflict of interest: Agata Tymińska, Krzysztof Ozierański, Marcin Grabowski, Grzegorz Opolski, Paweł Balsam: fees for lectures or participation in clinical trials: Novartis, Boehringer Ingelheim.

\section{References}

1. Savarese G, Lund LH. Global public health burden of heart failure. Card Fail Rev. 2017; 3(1): 7-11, doi: 10.15420/cfr.2016:25:2, indexed in Pubmed: 28785469.

2. Ponikowski P, Voors A, Anker S, et al. 2016 ESC Guidelines for the diagnosis and treatment of acute and chronic heart failure. Eur Heart J. 2016; 37(27): 2129-2200, doi: 10.1093/eurheartj/ehw128.

3. Ambrosy AP, Fonarow GC, Butler J, et al. The global health and economic burden of hospitalizations for heart failure: lessons learned from hospitalized heart failure registries. J Am Coll Cardiol. 2014; 63(12): 1123-1133, doi: 10.1016/j.jacc.2013.11.053, indexed in Pubmed: 24491689.

4. Balsam P, Ozierański K, Kapłon-Cieślicka A, et al. Differences in clinical characteristics and 1-year outcomes of hospitalized patients with heart failure in ESC-HF Pilot and ESC-HF-LT registries. Pol Arch Intern Med. 2019; 129(2): 106-116, doi: 10.20452/pamw.4418, indexed in Pubmed: 30648697.

5. Goldsmith SR. Interactions between the sympathetic nervous system and the RAAS in heart failure. Curr Heart Fail Rep. 2004; 1(2): 45-50, doi: 10.1007/s11897-004-0024-5, indexed in Pubmed: 16036024.

6. Fonarow GC, Stough WG, Abraham WT, et al. Characteristics, treatments, and outcomes of patients with preserved systolic function hospitalized for heart failure: a report from the OPTIMIZE-HF Registry. J Am Coll Cardiol. 2007; 50(8): 768-777, doi: 10.1016/j.jacc.2007.04.064, indexed in Pubmed: 17707182.

7. Kapłon-Cieślicka A, Tymińska A, Peller M, et al. Diagnosis, Clinical Course, and 1-Year Outcome in Patients Hospitalized for Heart Failure With Preserved Ejection Fraction (from the Polish Cohort of the European Society of Cardiology Heart Failure Long-Term Registry). Am J Cardiol. 2016; 118(4): 535-542, doi: 10.1016/j.amjcard.2016.05.046, indexed in Pubmed: 27374606.

8. Solomon SD, Rizkala AR, Gong J, et al. Angiotensin receptor neprilysin inhibition in heart failure with preserved ejection fraction: rationale and design of the PARAGON-HF trial. JACC Heart Fail. 2017; 5(7): 471-482, doi: 10.1016/j.jchf.2017.04.013, indexed in Pubmed: 28662936.

9. Novartis. A randomized, double-blind controlled study comparing LCZ696 to medical therapy for comorbidities in HFpEF Patients (PARALLAX). http://clinicaltrials.gov/ct2/show/NCT03066804. NLM identifier: NCT03066804 (accessed 20 December 2017).

10. Buggey J, Mentz RJ, DeVore AD, et al. Angiotensin receptor neprilysin inhibition in heart failure: mechanistic action and clinical impact. J Card Fail. 2015; 21(9): 741-750, doi: 10.1016/j.cardfail.2015.07.008, indexed in Pubmed: 26209000.

11. Yancy CW, Jessup M, Bozkurt B, et al. 2016 ACC/AHA/HFSA Focused Update on New Pharmacological Therapy for Heart 
Failure: An Update of the $2013 \mathrm{ACCF} / \mathrm{AHA}$ Guideline for the Management of Heart Failure: A Report of the American College of Cardiology/American Heart Association Task Force on Clinical Practice Guidelines and the Heart Failure Society of America. J Am Coll Cardiol. 2016; 68: 1476-1488.

12. Braunwald $\mathrm{E}$. The path to an angiotensin receptor antagonist-neprilysin inhibitor in the treatment of heart failure. J Am Coll Cardiol. 2015; 65(10): 1029-1041, doi: 10.1016/j.jacc.2015.01.033, indexed in Pubmed: 25766951.

13. Kaplinsky E. Sacubitril/valsartan in heart failure: latest evidence and place in therapy. Ther Adv Chronic Dis. 2016; 7(6): 278-290, doi: 10.1177/2040622316665350, indexed in Pubmed: 27803793.

14. von Lueder TG, Sangaralingham SJ, Wang BH, et al. Reninangiotensin blockade combined with natriuretic peptide system augmentation: novel therapeutic concepts to combat heart failure. Circ Heart Fail. 2013; 6(3): 594-605, doi: 10.1161/CIRCHEARTFAILURE.112.000289, indexed in Pubmed: 23694773.

15. McMurray J, Packer M, Desai A, et al. Angiotensin-neprilysin inhibition versus enalapril in heart failure. N Engl J Med. 2014; 371(11): 993-1004, doi: 10.1056/nejmoa1409077.

16. Velazquez EJ, Morrow DA, DeVore AD, et al. PIONEER-HF Investigators. Angiotensin-Neprilysin inhibition in acute decompensated heart failure. N Engl J Med. 2019; 380(6): 539-548, doi: 10.1056/NEJMoa1812851, indexed in Pubmed: 30415601.

17. Kristensen SL, Jhund PS, Mogensen UM, et al. Prognostic value of N-terminal pro-B-type natriuretic peptide levels in heart failure patients with and without atrial fibrillation. Circ Heart Fail. 2017; 10(10), doi: 10.1161/CIRCHEARTFAILURE.117.004409, indexed in Pubmed: 29018174.

18. Nessler J, Straburzyńska-Migaj E, Windak A, et al. [Expert consensus on the usefulness of natriuretic peptides in heart failure.]. Kardiol Pol. 2018; 76(1): 215-224, doi: 10.5603/KP.2017.0020, indexed in Pubmed: 29399772.

19. Diez M, Talavera ML, Conde DG, et al. High-sensitivity troponin is associated with high risk clinical profile and outcome in acute heart failure. Cardiol J. 2016; 23(1): 78-83, doi: 10.5603/ CJ.a2015.0058, indexed in Pubmed: 26412605.

20. Nakou ES, Marketou ME, Chlouverakis GI, et al. Troponin-I levels as a potential prognostic biomarker of sacubitril/valsartan treatment response in heart failure with reduced ejection fraction: Who will benefit most? Clin Cardiol. 2018; 41(12): 1548-1554, doi: 10.1002/clc.23099, indexed in Pubmed: 30324615.

21. Desai AS, Claggett BL, Packer M, et al. PARADIGM-HF Investigators. Influence of Sacubitril/Valsartan (LCZ696) on 30-Day Re- admission After Heart Failure Hospitalization. J Am Coll Cardiol. 2016; 68(3): 241-248, doi: 10.1016/j.jacc.2016.04.047, indexed in Pubmed: 27417000.

22. Novartis. Prospective ARNI vs. ACE inhibitor trial to determine superiority in reducing heart failure events after MI (PARADISE-MI). http://clinicaltrials.gov/ct2/show/NCT02924727. NLM identifier: NCT02924727 (accessed 29 December 2017).

23. Pascual-Figal D, Wachter R, Senni M, et al. Rationale and design of TRANSITION: a randomized trial of pre-discharge vs. post-discharge initiation of sacubitril/valsartan. ESC Heart Fail. 2018; 5(2): 327-336, doi: 10.1002/ehf2.12246, indexed in Pubmed: 29239515.

24. Wachter R, Senni M, Belohlavek J, et al. Sacubitril/Valsartan initiated in hospitalized patients with heart failure with reduced ejection fraction after hemodynamic stabilization: primary results of the TRANSITION study. J Cardiac Failure. 2018; 24(8): S15, doi: 10.1016/j.cardfail.2018.07.045.

25. Senni M, McMurray JJV, Wachter R, et al. Initiating sacubitril/ valsartan (LCZ696) in heart failure: results of TITRATION, a double-blind, randomized comparison of two uptitration regimens. Eur J Heart Fail. 2016; 18(9): 1193-1202, doi: 10.1002/ ejhf.548, indexed in Pubmed: 27170530.

26. Luo N, Lippmann SJ, Mentz RJ, et al. Relationship between hospital characteristics and early adoption of angiotensin-receptor/neprilysin inhibitor among eligible patients hospitalized for heart failure. J Am Heart Assoc. 2019; 8(3): e010484, doi: 10.1161/JAHA.118.010484, indexed in Pubmed: 30712431.

27. Seferovic PM, Ponikowski P, Anker SD, et al. Clinical practice update on heart failure 2019: pharmacotherapy, procedures, devices and patient management. An expert consensus meeting report of the Heart Failure Association of the European Society of Cardiology. Eur J Heart Fail. 2019; 21(10): 1169-1186, doi: 10.1002/ejhf.1531, indexed in Pubmed: 31129923.

28. Balsam P, Peller M, Borodzicz S, et al. In-hospital heart rate reduction and its relation to outcomes of heart failure patients with sinus rhythm: Results from the Polish part of the European Society of Cardiology Heart Failure Pilot and Long-Term Registries. Cardiol J. 2020; 27(1): 25-37, doi: 10.5603/CJ.a2018.0094, indexed in Pubmed: 30155862.

29. Cowie MR, Anker SD, Cleland JGF, et al. Improving care for patients with acute heart failure: before, during and after hospitalization. ESC Heart Fail. 2014; 1(2): 110-145, doi: 10.1002/ ehf2.12021, indexed in Pubmed: 28834628. 